
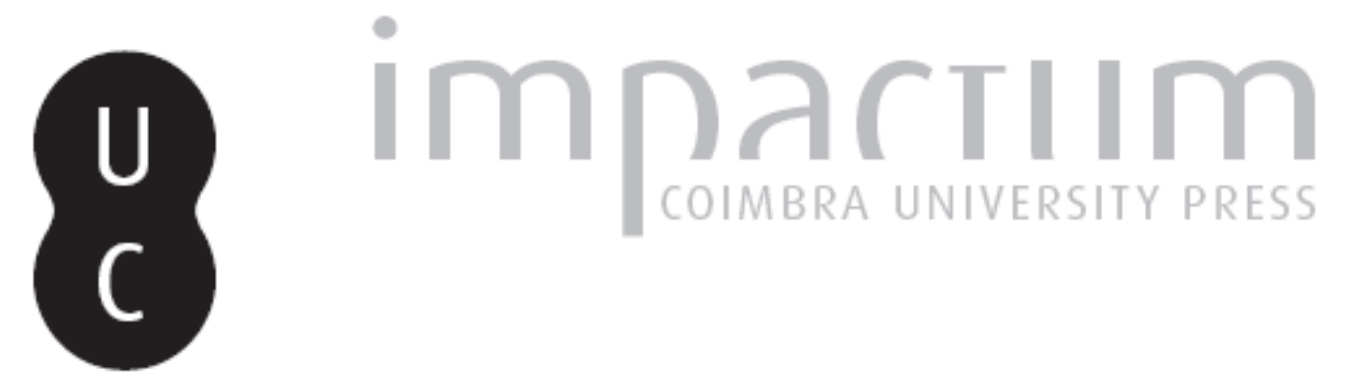

\title{
La darwiniana especie Homo sapiens
}

\section{Autor(es): Galera, Andrés}

Publicado por: CIAS - Centro de Investigação em Antropologia e Saúde

URL persistente:

URI:http://hdl.handle.net/10316.2/28629

DOI:

DOI:http://dx.doi.org/10.14195/2182-7982_27_3

Accessed : $\quad$ 26-Apr-2023 12:36:38

A navegação consulta e descarregamento dos títulos inseridos nas Bibliotecas Digitais UC Digitalis, UC Pombalina e UC Impactum, pressupõem a aceitação plena e sem reservas dos Termos e Condições de Uso destas Bibliotecas Digitais, disponíveis em https://digitalis.uc.pt/pt-pt/termos.

Conforme exposto nos referidos Termos e Condições de Uso, o descarregamento de títulos de acesso restrito requer uma licença válida de autorização devendo o utilizador aceder ao(s) documento(s) a partir de um endereço de IP da instituição detentora da supramencionada licença.

Ao utilizador é apenas permitido o descarregamento para uso pessoal, pelo que o emprego do(s) título(s) descarregado(s) para outro fim, designadamente comercial, carece de autorização do respetivo autor ou editor da obra.

Na medida em que todas as obras da UC Digitalis se encontram protegidas pelo Código do Direito de Autor e Direitos Conexos e demais legislação aplicável, toda a cópia, parcial ou total, deste documento, nos casos em que é legalmente admitida, deverá conter ou fazer-se acompanhar por este aviso. 


\section{Antropologia Portuguesa}

Volume $26-27 \cdot 2009-2010$

Departamento de Antropologia | Universidade de Coimbra

DARWINISMO:

revisitações, propostas, problemas 


\section{La Darwiniana especie Homo sapiens}

\section{Andrés Galera}

GEA, CCHS, CSIC

Proyecto HAR2009-12418, Espanha

andres.galera@cchs.csic.es

Resumen El artículo es una reflexión sobre el significado bio-antropológico que la especie Homo sapiens adquiere con la formulación de la teoría evolutiva darwiniana particularizada en los idearios de Thomas Huxley, Alfred Russel Wallace y el propio Darwin, desde la publicación de El origen de las especies hasta la aparición de El origen del hombre. El estudio analiza dichas variantes concluyendo que siendo modelos unidos por el principio de selección natural se diferencian claramente identificando filosofías singulares.

Palabras clave Darwin; evolución; Huxley; origen del hombre; Wallace.

Abstract This paper analyzes the bio-anthropological meaning that human species acquires with the formulation of Darwin's evolutionary theory following the ideology of Thomas Huxley, Alfred Russel Wallace and Darwin, from the publication of The origin of species to appearance of The descent of man. The research analyzes the three variants concluding that they are models linked by the natural selection and separated for own characteristics that identify them singularly.

Key words Darwin; descent of man; evolution; Huxley; Wallace.

Transcurrieron doce años entre la publicación del polémico On the origin of species $y$ The descent of man. Anticipándose a Darwin, en 1863 Thomas Huxley echaba leña al fuego publicando Evidence as to man's place in nature. El libro es la respuesta evolutiva por selección natural a una pregunta reiterada en la historia de la humanidad: ¿qué lugar ocupa el hombre en la naturaleza? Un sencillo interrogante para introducirnos en un problema complicado, descubrir qué es el fenómeno humano. Alcanzada la centuria de 1700 el enigma seguía subrogado al dogma religioso, la solución era aún fácil e inmediata, el Homo sapiens es la especie elegida por el creador para el uso y disfrute de los bienes terrenales. En el siglo XIX cambiaron las tornas, el tema ocupa un lugar en el árbol de la ciencia. Un colectivo de biólogos apuesta por la materia como elemento aglutinador de la vida suscribiendo 
la teoría evolutiva en detrimento de la teología natural. Subrayando el estereotipo diremos que el mono bajó del árbol para caminar erguido. Luego el homínido de turno aprende a controlar el fuego, a manipular los alimentos, comienza a independizarse del medio incrementándose exponencialmente las posibilidades de supervivencia. Una versión reducida de está historia la imaginó tempranamente un sagaz romano, Lucrecio Caro, en el poema De rerum natura; corrían los años 60 antes de Cristo. La fantasía literaria recrea una primigenia especie humana surgida de la dura tierra, y como ella de sólida constitución; cavernícolas vagando por bosques y praderas practicando el conocido juego de la subsistencia que, descubierto el fuego y simulando la acción del Sol sobre los frutos silvestres, aprenden a cocer y ablandar los alimentos (Caro, 1990: 303, 309); ahí comienza otra historia. La idea de un antepasado primitivo, salvaje, incivilizado, predecesor del hombre moderno no es un exclusivo atributo evolutivo y sí un legado cultural antiguo que la teoría de la evolución puso en valor aplicando el materialismo científico, dotando al hombre de una forma variable, colocándole en lugar y cronología particulares. Sea como fuere, sustituir el parentesco divino por un vínculo animal no es una opción atrayente aun siendo verdad. La hipótesis conlleva la renuncia a Dios para ocupar un puesto alternativo dentro de una naturaleza cambiante que no hace distingos con el hombre; situación igualitaria ideológicamente difícil de aceptar porque la humildad es una de las virtudes que menos nos representan. Sin embargo, los paleontólogos han sido implacables desenterrando la caterva de antepasados perdidos en el decurso evolutivo, obligándonos a aceptar con hechos consumados que la especie humana tiene su origen en un proceso de hominización ocurrido durante el Cenozoico: Sivaphitecus, Australopithecus, Paranthropus, Homo habilis, Homo erectus, Homo neanderthalensis, son los nombres de algunos productos evolutivos nacidos en el espacio transformista que nos amamantó, y no hay lugar para la duda.

Seguimos al reputado paleontólogo neodarwinista Geoge Gaylord Simpson al afirmar que fue el naturalista francés Lamarck quien valientemente dio forma a la idea de evolución para explicar el fenómeno de la vida terrestre (Simpson, 1967: 186), por consiguiente del hombre; lo hizo abiertamente el año 1809 en la consabida Philosophie zoologique, obra de título oscuro e ideas claras. Desde entonces la teoría forma parte de la comunidad científica suscitando un debate plural donde, pasadas cinco décadas, surge, toma forma y sentido, la darwiniana evolución por selección 
natural (Galera, 2002a; 2009a). En consecuencia, las superadas quinientas páginas del Origen componen un tratado biológico de tema conocido cuya novedad no consiste en reiterar la alternancia orgánica de seres vivos filogenéticamente emparentados, sí en proponer otra solución respecto al modo y a la manera de ocurrir dicha transformación. El Origen es un texto donde el omnímodo principio de la selección natural (Galera, 2009b), según el cual los individuos morfológicamente mejor adaptados triunfan en la lucha por vivir, gobierna la acción de la naturaleza, extinguiéndose y generando nuevas especies desde ayer hasta hoy y hacia mañana. En este universal, incesante, e inacabado proceso vital, el existir del Homo sapiens se suma a la teoría general, es otro punto y seguido en la secuencia animal terrestre, sobre cuya historia y origen se arrojará luz en el futuro, auguraba Darwin (1859: 488). Su correligionario Alfred Russel Wallace fue más concreto y atrevido. Narrando su viaje por el archipiélago Malayo, rememorando sus hazañas por la isla de Borneo cazando orangutanes despiadadamente, escribe que será en las tropicales cavernas y depósitos del terciario donde se hallen los restos necesarios para reconstruir la historia evolutiva de los monos antropomorfos y conocer a los antecesores de la actual fauna de gorilas, chimpancés, orangutanes, incluido el hombre (Wallace, 1869: 72); especies concurrentes en el tiempo, ancestralmente coincidentes, portadoras de un valor evolutivo propio. No juzgamos aquí lo acertado del pronóstico, sería ridículo atribuirle significado más allá de ser una conjetura ocurrente, recogemos el testimonio como ejemplo del cambio de mentalidad necesario para interpretar la condición humana atendiendo a la idea de evolución: principio rector del sistema natural desde la inicial génesis específica hasta los sucesivos desarrollos espacio-temporales.

La formulación decimonónica del concepto de evolución tuvo diferentes modalidades (lamarckismo, geoffroyismo, darwinismo, mendelismo, mutacionismo, ortogénesis, son algunas), convergentes en definir la vida terrestre como un suceso material cronológico desarrollado mediante la sustitución de unas especies por otras filogenéticamente emparentadas; y divergentes al explicar cómo y porqué ocurre este hecho biológico. En cualquiera de los casos, al aplicar la teoría de la evolución a la especie humana se generan sendas directrices cognitivas que vertebran el razonamiento: • una empírico-general, proyectada en la necesaria búsqueda paleontológica de extintos antepasados imbricados temporalmente certificando la realidad de la hipótesis planteada; • otra epistemológico-particular, otorgando a la especie 
Homo sapiens un estatus natural igualitario consecuencia de experimentar fenómenos análogos al resto de los seres vivos, de suerte que el diferente bagaje evolutivo no representa ningún atributo extra-sistémico, es sólo una propiedad emergente dentro del multiespecífico, aleatorio y transformista proceso diversificador de la vida, continuo en espacio y tiempo; acontecer verificado en modo de selección natural para el caso de la teoría darwinista. Sintéticamente, aceptar la evolución conlleva admitir un dictamen de casualidad respecto al ser anatómico que cada especie expresa con su particular fórmula vital, habiendo un marco de actuación común, único, para quienes habitan, habitaron y habitaran, la Tierra. Conocer al hombre iluminado por esta nueva dimensión científica sigue siendo una antropología inveterada, antigua, arraigada, dividida según la dicotomía kantiana de una antropología fisiológica, que lo visualiza como entidad biológica, más una antropología en sentido pragmático, que lo representa en su postrera faceta de individuo libre hacedor del mundo (Kant, 2004: 17); pero ambas experiencias son ahora anudadas por y trazadas desde el pensar evolutivo, ideario donde el fenómeno humano alumbra preguntas novedosas cuyas respuestas habrá que buscar, mientras que las viejas cuestiones tendrán soluciones nuevas. Arduo interrogatorio cuya finalidad es resolver esa duda mayúscula, intemporal, nominada por Darwin The descent of man.

Resulta requetesabido, para los evolucionistas claro está, que la semana del 30 de junio de 1860 la British Association for the Advancement of Science celebró en Oxford su meeting anual. Evolucionismo frente a creacionismo era el tema a trata. La reunión no defraudó. Cuentan las crónicas que ese sábado un público expectante abarrotó la biblioteca del Museo de Historia Natural habilitada como sala de conferencias. Thomas Huxley, apodado el bulldog de Darwin por su acérrima defensa de la doctrina, asistía al acto. El obispo Samuel Wilberforce, enemigo reconocido, era uno de los selectos participantes. El clérigo profirió sus ataques con ironía, elegancia, persuasión y sin fundamento científico, culminando su intervención preguntando al naturalista si descendía de un mono por parte de abuelo o de abuela; se armó la marimorena. Leyendo a Francis Darwin sabemos que en realidad era el paleontólogo Richard Owen quien movía los hilos entre bambalinas incitando al obispo para actuar contra su padre (Darwin, 1958: 250-1). No bastaron seis días, Huxley empleó alguno más, tres años, para redactar Evidence as to man's place in nature dando cumplida y pausada 
respuesta a la insolencia clerical: el hombre desciende del mono y ocurrió bien directamente por modificación gradual de una forma antropomorfa precedente, o colateralmente como rama separada de un primitivo tronco común (Huxley, 2006: 94). Unicidad de origen en ambos supuestos. Este hombre evolutivo es también un ser unitario con el todo animal, lo es en la composición y organización (Huxley, 2006) derivada de una fenomenología y materialidad continuadas durante el desarrollo de la vida desde lo inorgánico, efectivas en el devenir orgánico desde la inconsciencia de lo simple hasta la voluntad de lo complejo (Huxley, 2006: 96). Proceso donde encaja la evidente semejanza anatómica del hombre con los grandes simios antropomorfos (Huxley, 2006: 91), cuya distancia no es tanto por diferencias en la sustancia y en el contenedor formal como en la capacidad intelectual para procesar información mediante un sistema de comunicación verbal. Coincidiendo con el naturalista francés George Cuvier, el hombre evolutivo huxleyriano identifica a un Homo parlante cuya cualidad verbal, el lenguaje articulado, es la causa motriz de la separación evolutiva existente entre la humanidad y sus parientes cercanos (Huxley, 2006: 99). Almacenando conocimientos nacidos de la actividad individual, compartidos generación tras generación mediante el lenguaje intraespecífico, se forma un patrimonio cognitivo grupal ausente en las otras especies donde la información se pierde con la muerte individual (Huxley, 2006: 99); conjunto patrimonial a la vez efecto y causa de evolución pues una vez consolidado participa del proceso selectivo natural. Al hilo de esta valoración, implícito queda, y acertado, concluir que por mor de la evolución el Homo sapiens deja de ser un mera unidad anatómica estructuralmente modificable convertido también, y en mayúsculas, en un ser inteligente elaborador de información, cuya relación con el medio desemboca hacia un perpetuo proceso cognitivo uniendo herencia morfológica y herencia cultural como legado de la especie. Con el decurso evolutivo el fenómeno humano se complejiza mentalmente y la condición para sobrevivir da un giro copernicano ante la posibilidad de intervenir en la naturaleza modificándola favorablemente. Todo lo cual ocurre, y no podía ser de otra manera, en la dirección unívoca, e inequívoca, marcada por la selección natural. Sólo Darwin tiene razón, su teoría es la única capaz de dar sentido a los datos que la embriología, la anatomía comparada, la distribución geográfica, la paleontología, aportan sobre el origen y desarrollo de los seres vivos en general y del hombre en particular (Huxley, 2006: 94, 95). La radicalidad se hace obsesiva, el Homo sapiens 
puede alcanzar cotas insospechadas, siempre agarrado a la mano darwinista. Una autoritaria aplicación antropológica del principio de selección natural criticada públicamente, con énfasis particular por el tema de la unidad del género humano (Hunt, 1866).

En 1870 Alfred Russel Wallace publica Contributions to the theory of natural selection. El libro es una recopilación de trabajos elaborados entre los años 55 al 69, incluyendo algún inédito, remodelados, corregidos, ampliados para esta edición. En el elenco aparece "The development of human races under the law of natural selection", cuya precedente huella impresa se localiza en las páginas de Anthropological Review. Si compartiésemos la tesis expuesta deberíamos suscribir que el hombre no sólo culmina la naturaleza sino que ocupa un lugar aparte por constituir un ser nuevo y especial (Wallace, 1872: 340). ¿Cuáles son las claves de tan singular raciocinio? La primera reflexión es obligada, tan evidente como relegada históricamente. Wallace no practicó el seguidismo, llegó a la formulación del modelo evolutivo por selección natural de motu propio, simultánea e independientemente de Darwin, y estuvo capacitado para construir un horizonte sui generis transgresor del oficialismo. Estatus independiente desde el cual propuso una acción evolutiva diferente, una versión lamarckiana de la selección natural cuyo escenario es una naturaleza armónica, adaptativamente estable, donde los cambios orgánicos que transforman las especies ocurren por un desajuste en la relación individuo-medio. Las condiciones cambian y sólo los más aptos sobreviven iniciando un proceso selectivo continuado en el novedoso grupo reproductor que tal circunstancia origina, hasta alcanzar una nueva sinergia entre orgánico e inorgánico. Se podría concluir que la evolución es un ajuste anatómico del mundo vivo sintonizando la frecuencia medioambiental (Wallace, 1872: 326, 327). Alcanzado este punto, el caso del Homo sapiens representa, además, un cambio de tendencia en los parámetros evolutivos. Al manifestarse la vida animal como acto cooperativo se altera la dinámica selectiva. La particularidad física deja de ser la condición electiva, sustituida por aquellos valores comunitarios que favorecen la colaboración como mecanismo de supervivencia. Entonces, la tipológica permanece constante a resguardo de la actuación colectiva que permite adaptarse, sobrevivir, sin modificar el cuerpo (Wallace, 1872: 328-330). La opción desarrollada por la especie humana, donde la selección natural deviene selección social a favor de las variaciones mentales que aseguran el bienestar y la seguridad 
de todos. Dos etapas constituyentes definen la historia evolutiva del Homo sapiens. La primera, una época ancestral silvestre controlada evolutivamente por la selección morfológica; un estadio animal propio de un ser amoral e irreflexivo, con una capacidad intelectual reducida a la mera percepción. La segunda, un periodo vigente de civilidad condicionado por la selección social, caracterizado por la estabilidad tipológica tras la adquisición y el desarrollo de un cerebro facultado para actuar por sí mismo, para guiar los pasos del hombre con independencia del medio anulando la influencia de una selección natural remplazada por la actividad humana. El hombre evolutivo de Wallace es un Homo cerebral que discurre sobre los problemas y halla la manera de resolverlos; organización mental convertida en señal inequívoca de la transición homínida (Wallace, 1872: 331-8).

El Homo sapiens surge de un animal inferior sujeto a la misma materia y leyes que modifican al resto de animales pero su existencia tiene un valor añadido, significa que hay cosas que la selección natural no puede hacer (Wallace, 1872: 349). Al poner límites a la selección Wallace incurre en una clara contradicción ideológica viéndose obligado a reconducir el tema de manera acientífica. ¿Qué cosas son imposibles para la selección natural y por qué motivo? La inacción es operativa, viene determinada por la linde temporal inserta en el concepto de selección natural como mecanismo evolutivo. Regulado por los parámetros de competencia y utilidad adaptativa, el proceso selectivo únicamente tiene valor en el presente seleccionando lo que es útil en un momento dado bajo circunstancias concretas (Wallace, 1872: 351); carece de prospectiva de futuro más allá de ser el modelo a superar; conduce a una perfección relativa en espera siempre de algo mejor, hecho a considerar como un factor de inestabilidad sistémica. Cabe preguntar, ¿es esta la dinámica evolutiva adecuada para explicar el desarrollo cerebral humano y su deriva intelectual? La respuesta es categórica, no. No porque el cerebro es una apuesta de evolución futura. La presencia del órgano en homínidos y hombres primitivos no tendría utilidad directa, sí un valor potencial expresado posteriormente cuando, en otro tiempo y momento diferente, el Homo desarrolle la capacidad intelectiva inmanente transformándose en sapiens. Este desplazamiento temporal entre desarrollo morfológico y funcional excluiría al cerebro del radio de acción inmediato, característico y necesario, de la darwiniana selección natural (Wallace, 1872: 360). Wallace está en un callejón sin salida. El problema admite dos soluciones: aceptar que los seres vivos se ven afectados también por leyes evolutivas no darwinistas; 
admitir que existen fuerzas superiores, desconocidas, una inteligencia suprema tal vez, representando la voluntad universal, que dirigió la evolución humana hacia su culminación (Wallace, 1872: 350, 379, 389). La primera es una opción inaceptable, equivale a claudicar ideológicamente; la segunda, la elegida, es un caminar por las tierras movedizas de lo sobrenatural conduciendo la evolución hacia un pensamiento vitalista que la contemplará como manifestación de lo incognoscible. Posición defendida, verbigracia, por el reputado filósofo Henri Bergson en L'évolution créatrice, ya en el siglo $\mathrm{XX}$, bajo el concepto de élan vital. Aunque ni se busque ni se pretenda, el Homo sapiens cerebral es una figura parcialmente antidarwinista refutando la capacidad plena de la selección natural para guiar la evolución; razón fundamental por la que Charles Darwin no compartió el planteamiento de Alfred Wallace (Darwin, 1989: 54).

Confiesa Darwin en su Autobiografía que la escueta referencia al hombre incluida al final del Origen de las especies no fue un acto baladí sino el reconocimiento explícito de la implicación de la humanidad en la teoría general, al objeto de hacer patente dicho punto de vista. Durante años recopiló material sobre el tema, y en febrero de 1867 comenzó la redacción de un capítulo sobre el hombre a incluir en The variation of animals and plants under domestication. No fue así, el texto cobró vida propia convirtiéndose en The descent of man, publicado el 24 de febrero de 1871 con una tirada inicial de 2.500 ejemplares y 5.000 más finalizando el año (Darwin, 1958: 49, 287). En su primera parte, El origen del hombre es un recopilatorio de pruebas en favor de su descendencia a partir de alguna forma anterior e inferior. Camino trillado que sitúa al hombre en la cotidiana lucha por existir inmerso en el consabido proceso de selección natural. Todo responde al guión transformista trazado en el Origen que el género Homo escenifica con fidelidad soportando las mismas causas generales, leyes y fenómenos que gobiernan al resto de mortales; conjunción teórica derivada de facto de su coincidencia organizativa y estructural con el plan anatómico de los mamíferos (Darwin, 1989: 146). El corolario se repite, a pesar de su diferenciador bagaje moral e intelectual el hombre forma parte de un único sistema natural que aglutina a los seres vivos de cualquier época y lugar, lo lidera. Linneo tuvo, pues, razón al colocarlo presidiendo el orden de los primates (Darwin, 1989: 149); y ser darwinista supone un reencuentro con el extinto maestro. Reencuentro con función sistemática, 
fruto de responder la pregunta ¿qué es el hombre? con perspectiva zoológica, despojándolo de su nexo social, retrotrayéndolo a su estado animal: es el Homo sapiens, el primero de los monos (Linneo, 1758). Reencuentro con valor ideológico, aceptando el principio general linneado Natura non facit saltum, la naturaleza no da saltos, celebrado por el mismo Darwin como un hecho rigurosamente verdadero; un todo continuo con pleno sentido en su teoría evolutiva definiendo la relación espacio-temporal de los seres vivos en la sucesión geológica (Darwin, 1859: 206). De este reencuentro faltaban todavía muchas piezas paleontológicas por descubrir; de aquel Linneo salió vencedor del arduo conflicto anatómico mantenido contra el antropólogo Friedrich Blumenbach, en compañía del afamado naturalista Georges Cuvier, adoctrinando estos a favor de separar al hombre del orden de los primates por su condición bípeda frente al estatus cuadrumano de los grandes monos (Blumenbach, 1803: 66). La evolución lo implica y Huxley, no fue el único, lo demostró el orangután, el gorila, no sólo tienen pies sino que las semejanzas anatómicas con el hombre son más sorprendentes que las diferencias (Huxley, 2006: 82). Darwin lo imaginó, nuestro ancestro sería un arbóreo mamífero velludo, con rabo y puntiagudas orejas, habitante más que probable del continente africano (Darwin, 1989: 512, 155); lo que no pudo soñar fue que sus propios antepasados habitaron el nordeste de África hace 45.000 años, desplegándose posteriormente hacia Europa (The Genographic Project, 2010).

Alcanzada la década de los 70, la ascendencia homínida de la humanidad marca el debate antropológico evolutivo, y la altisonancia recae sobre la pluralidad racial generándose una duda razonable sobre su posible causa: ¿monogenismo o poligenismo? Respondiendo a la polémica, defendiendo la unidad de la especie, en la mente de Darwin el Homo sapiens deviene homo sexual. ¿Cuáles son los parámetros de la nueva propuesta? Entendida como elemento gestor de afinidades copulativas, la reproducción implica un control morfológico grupal al seleccionar qué individuos aportarán su genotipo a las futuras generaciones; es la selección sexual, proceso que habría resultado determinante en la formación de las razas humanas (Darwin, 1989: 192, 509, 511). Como variante de la selección natural, controlada por ella, la sexual se manifiesta en un ámbito propio y tiene un objetivo diferente logrado con similar método: la conformación tipológica, favorable ahora al apareamiento y distintiva de los sexos. Relatada en términos de animal sexuado, la historia evolutiva humana presenta hechos diferenciales fruto de una doble condición 
biológica: de un lado la selección natural, actuando como motor adaptativo en calidad de juez universal; por otro la selección sexual, favoreciendo la aparición de caracteres inútiles para sobrevivir, válidos sólo al aparearse. Una responde al valor general de sobrevivir; la otra al particular de trasmitir a la descendencia la información morfológica seleccionada, contexto que permite justificar la aparición de caracteres secundarios constitutivos de la diversificación racial conservando la unidad original (Darwin, 1989: 509). Un duplo lenguaje selectivo cuyas consecuencias invaden también la relación de género definiendo la evolución en masculino. A Darwin no le tiembla el pulso llegado el momento de proclamar la superioridad del hombre, capaz de ir más lejos que la mujer tanto a la hora de pensar, de razonar, de imaginar, como en la simple práctica manual (Darwin, 1989: 473); y no es una cuestión de tamaño cerebral sino de nivel evolutivo. La inferioridad femenina se asienta sobre una interpretación sui generis, restrictiva, del funcionamiento de las selecciones natural y sexual. Contemplada la historia evolutiva humana como la de un colectivo estructurado por la división de tareas al sector femenino no le correspondió pensar, desempeñó un papel sedentario, reproductor, básicamente dedicado al cuidado de la prole, contrapuesto al vigor y la inteligencia que el otro sexo practicaba inmerso en misiones de protección y búsqueda de alimento. Progresivamente, la selección perfeccionó ambas tipologías, acentuándose las carencias de uno y las virtudes del otro que la variante sexual hizo suyas individualizándolas como dimorfismo (Darwin, 1989: 473-4). La negación darwiniana es radical, e incluso reconociendo la superioridad femenina en facultades como la intuición, la percepción, la imitación, el juicio resulta contraproducente al entenderse tales habilidades como la manifestación de un comportamiento arcaico (Darwin, 1989: 473). La mujer es un personaje evolutivo capitidisminuido, sometido al liderazgo masculino incluso genéticamente al ser la figura paterna quien en la reproducción aportaría las cualidades intelectuales a los hijos compensando la limitación materna (Darwin, 1989: 474). La evolución deviene así un instrumento antropológico coercitivo propio de sociedades reguladas por la ley del más fuerte.

Al estudiar la evolución Huxley; Wallace y Darwin analizan la especie humana aplicando la lupa de la descendencia común controlada por la selección natural. Cada cual trazó su camino singular e irregularmente. Unos con devoción, otro con hostilidad hacia la causa, mostrando todos su peculiar forma de pensar el hombre como saber evolutivo. 


\section{Referencias bibliográficas}

Bergson, H. 1907. L'évolution créatrice. Paris, F. Alcan.

Blumenbach, F. 1803. Manuel d'histoire naturelle. Metz, Collignon.

Caro, L. 1990. La naturaleza. Madrid, Akal.

Darwin, C. 1859. On the origin of species by means of natural selection. London, John Murray.

Darwin, C. 1868. The variation of animals and plants under domestication. London, John Murray.

Darwin, C. 1871. The descent of man, and selection in relation to sex. London, John Murray.

Darwin, C. 1989. El origen del Hombre. Madrid, Edaf.

Darwin, F. (ed.) 1958. The autobiography of Charles Darwin and selected letters. New York, Dover.

Galera, A. 2002a. Modelos evolutivos predarwinistas. Arbor, 172(677): 1-16.

Galera, A. 2002b. Creating evolution. In: Puig-Samper, M. A.; Ruiz, R.; Galera, A. (eds.) Evolución y cultura. Madrid, Junta de Extremadura-UNAM-Doce Calles: 13-20.

Galera, A. 2007. El significado religioso de la teoría de la evolución. In: Polo, M. (ed.) Religión y ciencia. Cuenca, Universidad Castilla La Mancha: 111-126.

Galera, A. 2009a. Lamarck y la conservación adaptativa de la vida. Asclepio, 52(2): 129-140.

Galera, A. 2009b. La omnipresente selección natural. Éndoxa, 24(1): 47-60

Hunt, J. 1866. On the application of the principle of natural selection to anthropology, in reply to views advocated by some of Mr. Darwin's disciples. Anthropological Review, 4(15): 320-340.

Huxley, T. 1863. Evidence as to man's place in nature. London, Williams and Norgate.

Huxley, T. 2006. Evidence as to man's place in nature. New York, Barnes \& Noble. Kant, I. 2004. Antropología. Madrid, Alianza.

Linneo, C. 1758. Systema naturae. 10. ${ }^{\mathrm{a}}$ ed. Estocolmo, Laurenti.

Simpson, G. G. 1967. La vida en el pasado. Madrid, Alianza.

The Genographic Project. 2010. Discovering the origins of Charles Darwin. [online]. Washington D.C., National Geographic. [Accessed 5-4-2010]. Available at: https:/genographic.nationalgeographic.com/genographic/lan/en/darwin.html.

Thomson, K. S. 2000. Huxley, Wilberforce and the Oxford Museum. American Scientist, 88(3): 210. 
Wallace, A. R. 1869. The Malay archipelago. New York, Harper \& Brothers.

Wallace, A. R. 1870. Contributions to the theory of natural selection. London, Macmillan.

Wallace, A. R.1872. La sélection naturelle, essais. Paris, Reinwald.

Wilberforce, S. 1860. Review of "On the origin of species" 1860. Quarterly Review, 108(215): 225-264.

Artigo recebido a 5 de Abril de 2010 e aceite a 23 de Julho de 2010. 